\title{
A Proposal of Wireless Network Routing Protocol for Heterogeneous Mobility
}

\author{
Tomohiko YAGYU ${ }^{\dagger}{ }^{\dagger \dagger}$, Student Member, Masahiro JIBIKI ${ }^{\dagger \dagger}$, \\ and Kenichi YOSHIDA ${ }^{\dagger}$, Members
}

\begin{abstract}
SUMMARY
Most wireless networks consist of heterogeneous nodes with diverse characteristics. These heterogeneous nodes have various moving characteristics such as speed and pausing time. Since conventional wireless routing schemes are designed for networks with homogeneous mobility, it is difficult to accomplish communication without degrading its quality, e.g., packet reachability and delay, in networks with heterogeneous mobility. In this paper, we propose efficient extensions of a proactive routing protocol to achieve sufficient communication quality in networks with heterogeneous mobility. The proposed extensions consist of three features, i.e., differential topology update, unidirectional movement notification and link quality based route calculation. Complementary actions among these functions can improve communication quality with acceptable control overhead. Simulation results reveal that the proposed scheme can achieve higher packet reachability and lower delay with low control overhead compared with existing routing schemes.

key words: Wireless network, Mobile Ad Hoc Network, Routing protocol, Heterogeneous Mobility
\end{abstract}

\section{Introduction}

Mobile ad hoc network (MANET) is an efficient technology for providing a wide-area communication environment where installing the infrastructure of a wired network is difficult. It is also suitable for supporting communication among mobile nodes. For the last several decades, many routing protocols have been proposed to make the best use of wireless network technology [1][2][3]. These routing schemes are classified into three categories, i.e., proactive (table-driven), reactive (on-demand), and position-based.

Among them, proactive routing is known to be the best scheme for interconnection with wired networks such as the Internet. This is because proactive scheme is based on the same principle as existing Internet routing protocols such as RIP [4] and OSPF [5]. It is important to interconnect with the Internet or wired backbone network for many MANET applications. Furthermore, since proactive scheme can provide us the ex-

Manuscript received January 1, 2003.

Manuscript revised January 1, 2003.

Final manuscript received January 1, 2003.

${ }^{\dagger}$ The authors are with the Graduate School of Business Science Tsukuba University, Otsuka Bunkyo-ku Tokyo 1120012 Japan

${ }^{\dagger \dagger}$ The authors are with the System Platforms Research Laboratories NEC Corporation, Nakahara-ku Kawasaki-shi Kanagawa 211-8666 Japan act information about all nodes joining MANETs, it is easy to connect multiple MANETs via wired backbone network. Considering these characteristics of proactive scheme and taking advantage of them, our research focuses on the proactive routing scheme.

One of the target applications of wireless ad hoc networks that we have in mind is a rescue operation in a disaster area. In such environments, wireless communication equipments carried by workers have different movement characteristics, such as speed and pausing time, from those mounted on vehicles. Communications will frequently occur between leaders in vehicles and workers on foot. They may also communicate with other parties through the Internet or wired backbone networks. One of the most important properties for such applications is the communication quality, e.g., packet reachability and delay. Because most existing proactive protocols are designed for networks with homogeneous mobility, it is difficult to adapt them to real networks with heterogeneous mobility. Although some protocols can work well by careful selection of optimal parameters for each environment, a slight change of the environmental conditions can easily spoil the optimal parameters.

In this paper, we propose efficient extensions for a proactive routing protocol, Scalable Mobility Adaptive Routing Techniques (SMART) that achieves better communication quality with low control overhead for mobile ad hoc networks with heterogeneous movement characteristics. SMART is based on Optimized Link State Routing (OLSR) [6], one of the standardized proactive MANET routing protocols in IETF [8]. SMART has the following three features beyond those of original OLSR.

\section{Differential topology update:}

To update topology information more quickly, SMART advertises only differential information about network topology after a link status changes with random postponements.

Unidirectional movement notification:

SMART focuses on the reduction of total control overhead to support heterogeneous mobility in the network. To reduce the overhead, SMART introduces unidirectional movement notification mechanism (we call it UMN for short later). UMN has 
two features. First, it has indirect link break detection function. This function can suppress constant message exchange and decrease the overhead to detect link status changes. Second, it has a new message generation mechanism for differential topology update. This mechanism can reduce a number of control messages.

\section{Link quality based route calculation:}

To select better routes before a route breaks, SMART utilizes link quality information for route calculation. All nodes in the network globally share the link quality information.

The rest of this paper is organized as follows. Section 2 provides an overview of the related works. Section 3 provides the details of our proposed routing techniques. Section 4 presents simulation results and considerations regarding performance evaluation. Finally we summarize our contributions in Section 5.

\section{Related Works}

OLSR [6] is one of the popular routing protocols for MANET. OLSR is based on a link state routing scheme like that of OSPF [5]. An OLSR node periodically broadcasts a Hello message to discover its neighbor nodes and establish local links. In addition, it periodically advertises its link information by flooding a Topology Control (TC) message over the network to establish global connectivity. The outstanding feature of OLSR is its method for flooding control packets by using the multi point relay (MPR) nodes. To reduce the overhead for flooding control packets over the network, each node selects a portion of its neighbor nodes as MPRs. Only neighbor nodes selected as MPRs must generate TC messages and forward the control packets received from selecting nodes. Refraining mechanism of forwarding nodes drastically reduces the number of control packets. Although OLSR has sufficient routing capability for homogeneous wireless networks, it leaves some room to improve for heterogeneous mobility. Since OLSR requires constant message intervals, it can not achieve sufficient reachability with acceptable overhead in networks with heterogeneous mobility.

There are two typical approaches to improve the packet reachability of routing protocols. The first is a combination of quick detection of topology changes (link establishment and breakage) and timely updating of topology information. Another is soft handover, i.e., smooth switching to better routes instead of using a degrading current route.

Benzaid et al. proposed extensions of OLSR, specifically "fast-OLSR" [9][10], to achieve quick detection of link changes with moving nodes. While FastOLSR nodes move faster than a threshold speed, they send fast-Hello messages at short intervals to detect link breakages quickly. Fast-Hello message includes only
MPR node information, and all neighbors selected as MPRs must reply with empty fast-Hello messages at the same short interval. Fast-OLSR nodes advertise its link status changes immediately by flooding a TC message. To reduce the control overhead in a fastOLSR network, the number of moving nodes' MPRs is limited to a fixed small number. Since the MPR selection method for the moving nodes does not take flooding accuracy into account, fast-OLSR may choose insufficient or redundant MPRs. Although the experimental results reported in [9] showed that fast-OLSR can control routing for a simple moving pattern efficiently, we found that neighbors caused heavy control overhead and it can not achieve sufficient communication quality in more complex situations (See Section 4 for details). Moreover, the restriction on the number of MPRs sometimes divides the network. Fast-OLSR seems to have been designed under the assumptions that moving nodes move straight as does a car on a highway and that they do not forward any packets. Therefore, this technique cannot handle complex mobility situations such as rescue operations.

Current cellular networks and wireless LAN Access Points have the ability of soft handover based on physical layer properties such as signal strength or bit error rate [11]. To improve packet reachability, we propose the use of these soft handover mechanisms into the MANET proactive routing scheme in this paper. One of the significant concerns for this technique is increase of control messages to advertise such link quality information. There are two approaches to reduce the control overhead. One is the differential update introduced in TBRPF [12]. Another is the hierarchical routing scheme, e.g., HSR [13] and LANMAR [14]. Because heterogeneous mobility is likely to damage a hierarchical network structure, we think that differential update is more suitable than the hierarchical approach for our target networks. So we make use of the differential update mechanism for the proposed method in this paper.

\section{Proposed Techniques: SMART}

Using OLSR and fast-OLSR, we could not achieve the sufficient communication quality, e.g., packet reachability or delay, in our simulation of networks with heterogeneous mobility (See experimental results shown in the next session). The following factors are the main reasons for degraded quality.

- Slow detection of link establishment and breakage.

- Slow switching of routes after a link breakage occurs.

- Frequent packet collisions and interference due to the heavy control overhead.

Therefore, we were motivated to develop a routing protocol for better communication in the networks with heterogeneous mobility. To achieve better packet 
reachability, SMART accomplishes two essential functions, i.e., quick detection of link changes and quick route switching before a route breaks. Furthermore, to prevent the increase of packet delay, we designed SMART to reduce the control message overhead for link maintenance and topology advertisement.

\subsection{SMART Messages}

SMART introduces three new messages: the MN (Movement Notification) message, the MR (Movement notification Reply) message and the TU (Topology Update) message. A moving node sends the MN message including its own ID while it moves faster than a threshold speed. By receiving MN messages, neighbor nodes can detect new links with the moving node. They can also detect link breakage by the expiration of validity time of MN message. The MN message also includes neighbor node IDs from which a moving node receives recent MR messages. The MR message is sent by the neighbor nodes of a moving node to establish a local link between them. It includes node IDs of moving nodes with which the neighbor node try to establish a local link ${ }^{\dagger}$. The TU message includes the link information that consists of node ID, link status (SYM or LOST) and link quality that is described in the following subsection. The TU message is used to advertise the link status and quality changes caused by the movement of fast moving nodes. SMART introduces differential topology updates by using the TU message. The TC message is also extended to have link status and link quality information. Table 1 summarizes the control messages used for SMART.

Table 1 Control messages for SMART

\begin{tabular}{|l|l|}
\hline Type & Description \\
\hline Hello & $\begin{array}{l}\text { Hello was originally introduced by OLSR to establish } \\
\text { local links with neighbors. In SMART, it is extended to } \\
\text { include the link quality and node's position information. }\end{array}$ \\
\hline TC & $\begin{array}{l}\text { TC was originally introduced by OSLR to advertise } \\
\text { global link connection periodically. In SMART, it is } \\
\text { also extended to advertise the link quality. }\end{array}$ \\
\hline MN & $\begin{array}{l}\text { MN is newly introduced for SMART to keep local con- } \\
\text { nectivity with fast moving nodes. It includes position } \\
\text { information and neighbor node IDs with which the mov- } \\
\text { ing node try to establish local links. }\end{array}$ \\
\hline MR & $\begin{array}{l}\text { MR is newly introduced for SMART to respond to MN } \\
\text { messages from neighbor nodes. It includes the ID of } \\
\text { moving nodes from which the neighbor nodes received } \\
\text { MN messages. }\end{array}$ \\
\hline TU & $\begin{array}{l}\text { TU is newly introduced for SMART to advertise the } \\
\text { changes of link status and quality of moving nodes glob- } \\
\text { ally. It has updated link information including node ID, } \\
\text { link status, and quality. }\end{array}$ \\
\hline
\end{tabular}

${ }^{\dagger}$ As in the conventions of OLSR, we call an established local link a symmetric (SYM) link.

\subsection{Unidirectional Movement Notification}

In the networks with heterogeneous mobility, it is an efficient technique to detect the link status changes that moving nodes send some messages at short interval. Fast-OLSR uses this technique as its fast-Hello message mechanism. Differential topology update is also efficient to advertise topology changes over the network. Fast-OLSR also introduces differential topology update mechanism. However, fast-OLSR could not achieve sufficient communication quality for the networks with heterogeneous mobility. One of the reasons is its heavy control overhead. Since heavy control overhead causes frequent packet collisions in wireless networks, packet reachability and delay are degraded. To decrease the overhead of bidirectional message exchange for fastHello, fast-OLSR omits information which is required to select optimal MPRs. Since a moving node cannot select adequate MPRs, fast-OLSR generates redundant TC messages.

SMART introduces unidirectional movement notification mechanism (UMN) to overcome these drawbacks of fast-OLSR. UMN was designed to reduce total control overhead with keeping effects of fast-Hello and differential topology update for heterogeneous mobility. We focused on two overhead of fast-OLSR, i.e., bidirectional fast-Hello exchange and redundant TC messages. SMART introduces indirect link break detection mechanism to avoid bidirectional message exchange. And to suppress redundant TC messages, SMART introduces different TU message generation mechanism. Since the selection of optimal MPRs by moving node is essential to flood TU messages, it is also necessary to introduce triggered Hello for SMART. In the following subsections, we explain the detail of each function.

\subsubsection{Indirect link break detection}

SMART avoids constant Hello message exchange between moving nodes and their neighbors. When a node starts moving beyond a threshold speed, it starts sending MN message at a short interval. The MN and MR message are used to establish new local links between moving nodes and their neighbors. Only neighbor nodes that do not have a local link with the moving node reply with an MR message to establish a local link. Since no neighbors reply with messages to the moving node if they already have a local link with it, the moving node does not constantly receive any messages from its neighbors. Moving nodes and neighbor nodes can establish local links by exchanging MN and MR messages only once.

The drawback of neighbor nodes' reply suppression is that the moving node can not detect the link breaks with its neighbors. To overcome this drawback, SMART makes use of the topology update message. 
When a neighbor node detects a link break by the expiration of valid MN message, the neighbor node sends a TU message to advertise the lost link. By receiving the TU message via other neighbors, the moving node can recognize that the link with the neighbor has broken.

3.2.2 Message generation mechanism for differential topology update

To reduce the overhead for differential topology update, SMART adopts different criteria of message generation from fast-OLSR. TU message is generated

- by a moving node only when it established a new link with its neighbors.

- by a neighbor node when it detected a link break with a moving node due to the time-out of MN message.

- by a neighbor node when it detected a change of link quality with a moving node. ${ }^{\dagger}$.

To select MPRs optimally and flood the TU messages with optimal MPRs, triggered Hello messages are sent in the following conditions.

1. When a node established a new link by receiving a MR message, and this causes the change of its MPRs.

2. When a node established a new link by receiving a MN message.

3. When a node detected a link breakage by the expiration of MN message, and this causes the change of its MPRs.

This triggered Hello sends full neighbor information which is not included in MN, MR and fast-Hello messages. Therefore, the triggered Hello enables the moving node to select optimal MPRs. TU messages are not generated by non-optimal MPRs. Furthermore, the message size is reduced by the TU message which includes only differential information.

\subsection{Link quality based route calculation}

If a node depends only on a time-out scheme to detect a change of link status, routes breaks frequently cause packet losses. To avoid such packet losses, a node should switch to a better route before a break in an active route occurs. SMART introduces link quality information so that a node can switch routes before the potential break. SMART has three levels of link quality, i.e., Fine, Degraded and Lost. The link quality is determined by distance between the nodes when they receive Hello and MN messages. The MN and Hello message includes the node's position information obtained from GPS or other devices. The Hello message

\footnotetext{
${ }^{\dagger}$ Link quality is explained in the next subsection
}

of SMART also includes the Rx link quality of each neighbor node. The Rx link quality is determined as follows.

$$
q_{R x}=\left\{\begin{array}{cl}
\text { Degraded } & \left(d_{t 1}<d\right) \\
\text { Degraded } & \left(d_{t 2}<d \leq d_{t 1} \wedge\right. \\
\text { current quality }=\text { Degraded }) & \left(d_{t 2}<d \leq d_{t 1} \wedge\right. \\
\text { Fine } & \text { current quality } \neq \text { Degraded }) \\
\text { Fine } & \left(d \leq d_{t 2}\right)
\end{array}\right.
$$

where $q_{R x}$ is $\mathrm{Rx}$ link quality, $d$ is a distance between nodes calculated from the position information in Hello or MN message. Two thresholds are introduced to prevent the oscillation of the link quality status. $d_{t 1}$ is the threshold of distance for quality degradation, $d_{t 2}$ is the threshold for quality recovery. Instead of distance information, received signal strength can be used to decide the $\mathrm{Rx}$ link quality. It may be more practical for the environments with many obstacles. However, we use the distance information for simulation simplicity.

The Tx link quality is also estimated from distance information calculated by the position information in $\mathrm{MN}$ and Hello messages. When a node receives the MN message, it determines the Tx Link quality as follows.

$$
q_{T x}=\left\{\begin{array}{cl}
\text { Lost } & \left(d_{\max }<d\right) \\
\text { Degraded } & \left(d_{t 1}<d \leq d_{\max }\right) \\
\text { Degraded } & \left(d_{t 2}<d \leq d_{t 1} \wedge\right. \\
& \text { current quality }=\text { Degraded }) \\
\text { Fine } & \left(d_{t 2}<d \leq d_{t 1} \wedge\right. \\
\text { Fine } & \left(d \leq d_{t 2}\right)
\end{array}\right.
$$

where $q_{T x}$ is Tx Link quality and $d_{\max }$ is the maximum distance of radio transmission range. When a node receives a Hello message, it can obtain its Tx Link Quality from the Rx Link quality of its own link in the Hello message. The total link quality is determined from $\mathrm{Rx}$ and Tx Link quality according to Table 2.

Table 2 Link quality determination mechanism

\begin{tabular}{|c||c|c|c|}
\hline$q_{R x} / q_{T x}$ & Fine & Degraded & Lost \\
\hline \hline Fine & Fine & Degraded & Lost \\
\hline Degraded & Degraded & Degraded & Lost \\
\hline
\end{tabular}

The neighbor nodes of the moving node advertise the changes in link quality by sending a TU message after random postponement. This random postponement prevents control message collisions. Receiving TU message triggers recalculation of the routing table. The following sequence is used to calculate the routes.

1. First, a node calculates the shortest routes to all nodes using only Fine links. 
2. If the first step fails to construct routes to some nodes, the node calculates routes to those nodes using all valid links.

\subsection{Message sequence example}

Fig.1 shows an example of message sequences of SMART and fast-OLSR. The top sequence in the figure is the message sequence of SMART and the bottom one is that of fast-OLSR. In this SMART example, node B and moving node $\mathrm{A}$ have a local link connection before node A starts moving. Node $\mathrm{C}$ and moving node $\mathrm{A}$ do not have a local link. After node $\mathrm{C}$ receives a $\mathrm{MN}$ message from moving node $\mathrm{A}(\mathrm{t}=\mathrm{t} 1)$, node $\mathrm{C}$ tries to establishes a new local link with moving node $\mathrm{A}$ by sending the MR message. After node B loses connection with moving node $\mathrm{A}(\mathrm{t}=\mathrm{t} 2)$, the time out of the MN message from node A causes node $\mathrm{B}$ to send a TU message that advertises the lost connection with node A. Moving node A receives the TU message from node $\mathrm{B}$ through node $\mathrm{C}$ and recognizes the fact that it lost the connection with node B. In contrast, in the fast-OLSR message sequence, node $\mathrm{B}$ and node $\mathrm{C}$ reply with fast-Hello whenever they receive a fast-Hello from moving node A. Therefore, fast-OLSR causes high Hello message overhead proportional to the number of MPRs.

\section{Performance Evaluation}

\subsection{Simulation Environment}

According to an activity report of the international rescue team for the Taiwan earthquake in 1999 [15], 110 members were engaged as a team in the operation. They were divided into three groups and each group had a truck and a bus. Since the team leader had a micro bus, seven vehicles were used in total. In addition, a helicopter was used to transfer team members and commodities to isolated areas. They used transceivers, cellular phones, the INMARSAT, and satellite mobile phones (IRIDIUM) for the communication with each other and a rear base in Japan. The kind of communication difficulties they encountered in this situation are also reported in [15].

In the experiments reported in this section, we assume a situation similar to the same kind of rescue operation as that in a disaster area of Taiwan. In our scenario, a large number of workers cooperate to rescue the injured, to dismantle destroyed buildings, and to build temporary dwellings. Several vehicles such as ambulances, fire engines, trucks and buses are used in the field. The communication between leaders (team leader and group leaders) and members will be most important and frequent. The leaders will often move by vehicles. Most communication will be transmission of voice streams. It sometimes will be other data, for example, as texts and photos.

With such a scenario in mind, we have assumed small numbers of high mobility nodes (i.e., leaders) and large numbers of low mobility nodes (i.e., members) in a limited field. We assumed the total number of nodes to be 100, and that 5 nodes would move as fast as vehicles. The other 95 nodes would move as slowly as pedestrians. The movement of nodes followed a random-waypoint mobility model. The speeds of low mobility nodes were randomly chosen between $1 \mathrm{~m} / \mathrm{s}$ to $2 \mathrm{~m} / \mathrm{s}$. The pause times of low mobility nodes were randomly chosen between 10 to 30 seconds. The speed of high mobility nodes was set to be $5,10,15$ and $20 \mathrm{~m} / \mathrm{s}$. The pause times of high mobility nodes were randomly chosen between 10 to 120 seconds. Each node was assumed to be equipped with an IEEE 802.11b wireless interface with 11 Mbps of bandwidth. The radio transmission range was assumed to be $200 \mathrm{~m}$ and the simulation field was set as 1000 meters square. The data packets were transferred bidirectionally between low mobility nodes and high mobility nodes. The source and destination nodes were randomly selected.

To emulate voice communication, the data traffic was assumed to have constant bit rate (CBR). The data rate was set to be $16 \mathrm{Kbps}$ in each direction (32 Kbps for one connection). The interval between traffic generations followed an exponential distribution with average of 60 seconds. The duration of the connection followed an exponential distribution with average of 180 seconds. On average, three connections were to be carried on in parallel.

\subsection{Simulation parameters}

We examined the performance of original OLSR, fastOLSR and SMART by simulations. We made use of OOLSR [17] in NS-2 [16]. Fast-OLSR and SMART were implemented based on OOLSR. We performed simulations for 10 scenarios with different mobility and traffic pattern, and took an average of results for all scenarios. Each simulation ran for 600 simulated seconds. We compared the following 5 variations.

- original OLSR (Hello Int. $=2 \mathrm{sec}, \mathrm{TC}$ Int. $=5 \mathrm{sec}$ )

- original OLSR (Hello Int. $=1 \mathrm{sec}, \mathrm{TC}$ Int. $=5 \mathrm{sec}$ )

- original OLSR (Hello Int. $=5 \mathrm{sec}$, TC Int.$=12.5 \mathrm{sec}$ )

- fast-OLSR (Hello Int. $=5 \mathrm{sec}$, TC Int. $=12.5 \mathrm{sec}$ )

- SMART (Hello Int. $=5 \mathrm{sec}$, TC Int. $=12.5 \mathrm{sec}$ )

We used the default values in RFC [7] for the Hello interval and TC interval. They are 2 seconds and 5 seconds respectively. We also examined shorter and longer intervals for original OLSR. Since the default intervals caused fast-OLSR and SMART to have too much control overhead, the intervals for the fast-OLSR and SMART were set to be the same as the longer intervals for original OLSR. The intervals of fast-Hello 


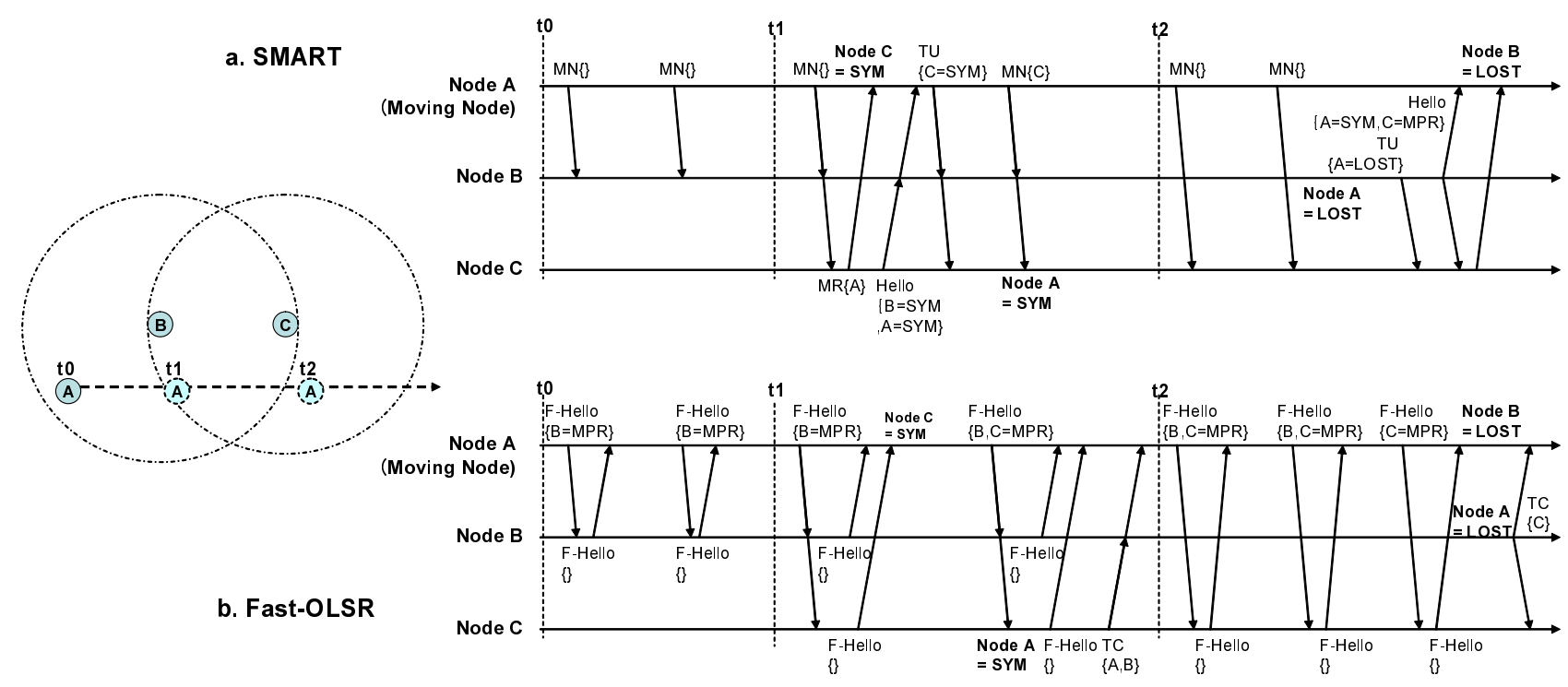

Fig. 1 Comparison of message sequences of SMART and fast-OLSR

for fast-OLSR and MN message for SMART were both set to be 1 second. The maximum number of MPRs for fast-OLSR was set to be 4 . Other parameters for simulations are shown in Table3.

Table 3 Simulation parameters

\begin{tabular}{l|c}
\hline parameter & value \\
\hline Threshold of speed for Movement notification & $5(\mathrm{~m} / \mathrm{sec})$ \\
Max Transmission range $\left(d_{\max }\right)$ & $200(\mathrm{~m})$ \\
Distance threshold for quality degradation $\left(d_{t 1}\right)$ & $180(\mathrm{~m})$ \\
Distance threshold for quality recovery $\left(d_{t 2}\right)$ & $170(\mathrm{~m})$ \\
\hline
\end{tabular}

\subsection{Simulation Results}

Fig.2 shows the packet reachability of each method. SMART outperformed original OLSR and fast-OLSR. The packet reachability of SMART, over $90 \%$, is sufficient to support VoIP communication. Fast-OLSR contrary made no improvement compared with original OLSR with same intervals. This result confirms that fast-OLSR is not suitable for free movement. Fig.3 shows the reasons for packet losses. This shows that most drops were caused by the failure of ARP (IFQ$\mathrm{ARP}$ ) and the excess of MAC retransmission limit (MAC-RET). Both failures happened in following two situations.

- Wrong route : A node tried to forward packets to a wrong next hop node, because updating routes is too slow.

- Collisions : Packets are broken or not sent by collisions with other node's transmission.

Therefore, we can say that SMART can rapidly switch

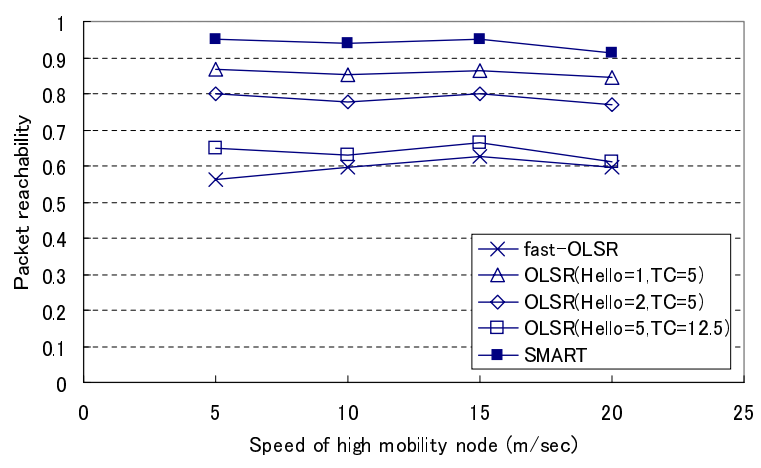

Fig. 2 Packet reachability

the routes with less collisions. With fast-OLSR, more packets are dropped by absence of valid routes (RTRNRTE) than with other methods. This is because TC message losses caused by collisions or incomplete MPR flooding make route calculation fail. SMART caused routing loop (RTR-LOOP) and TTL expiration (RTRTTL). There are two reasons for that.

- The moving node indirectly detects a link breakage with its neighbor by TU message. It causes time lag for route recalculation.

- SMART nodes calculate routes using link quality information. TU message loss causes mismatch of link quality information.

However, it does not seriously harm the total reachability. Original OLSR with shorter interval achieves better packet reachability than other original OLSR. This means that the overhead of frequent Hello message causes less effect on the packet reachability than TC message overhead.

Fig. 4 shows the average of control overhead. Here, 


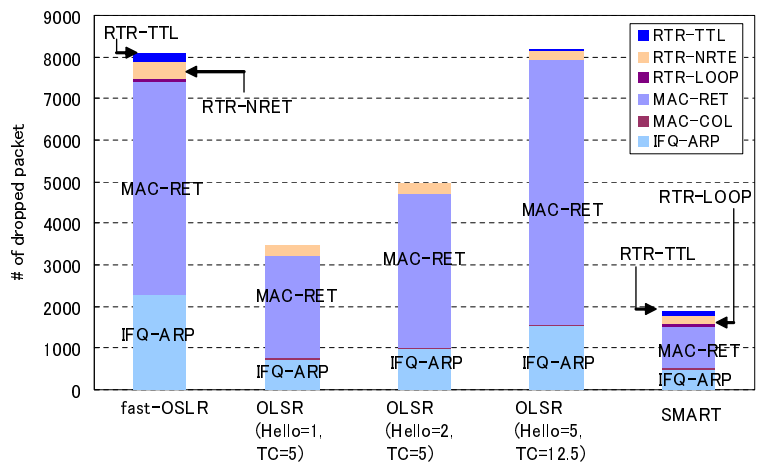

Fig. 3 Histogram of reasons for packet drop

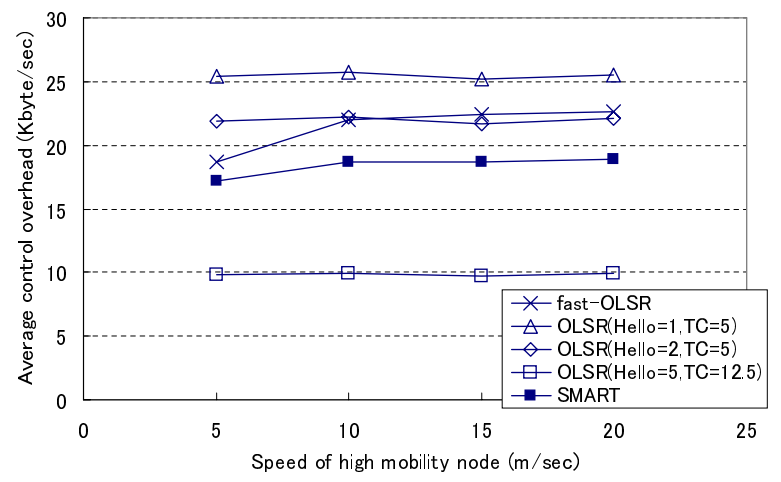

Fig. 4 Average control overhead

control overhead means the total byte size of routing packets generated in the network per second. This result shows the fact that the total control overhead of SMART is lower than fast-OLSR and original OLSR with default and shorter intervals.

Fig.5 compares control overhead in detail. Since original OLSR exchanges full Hello messages constantly, its Hello message's overhead is 3 times larger than that of others. Furthermore, because of triggered Hello messages, SMART's overhead of Hello, MN and MR messages are about 30\% larger than those of fast-OLSR. However triggered Hello enables SMART to maintain optimal MPRs. With optimal MPRs, SMART generates less overhead for topology update. It is about $18 \%$ less than fast-OLSR. As a whole, SMART can reduce total control overhead by $14 \%$ on average from fast-OLSR.

Unidirectional movement notification mechanism comprises several functions. Indirect link break detection function can mitigate the overhead of triggered Hello messages. And triggered Hello messages contribute reduction of TU message generation. New message generation mechanism for topology update suppresses redundant TU message. Total overhead of SMART can be reduced by the integrated effects of these functions.

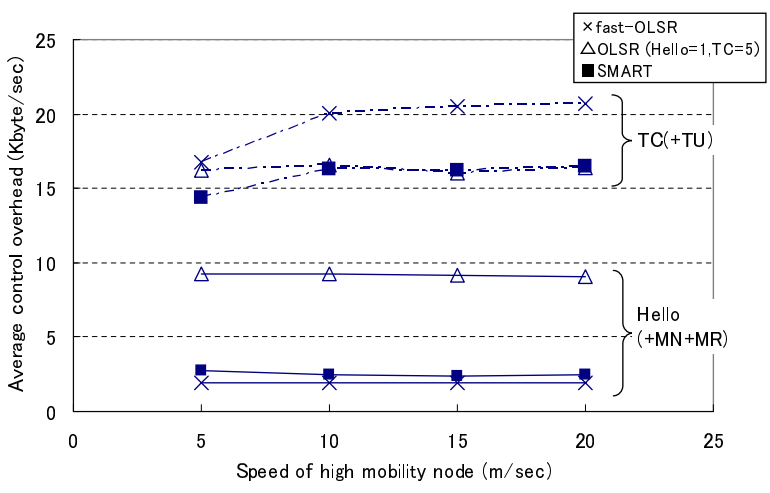

Fig. 5 Detail of overhead

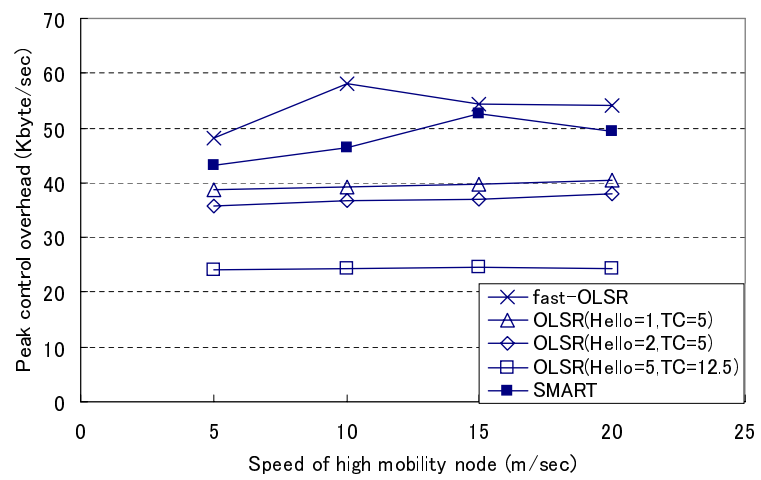

Fig. 6 Peak control overhead

Fig. 6 shows the peak control overhead. Peak control overhead means the maximum total size of control messages generated in the network per second. Unlike original OLSR, differential topology update mechanism in fast-OLSR and SMART makes control messages dynamically. The more control messages happens, the more packet collisions will occur. Therefore, it is important to reduce the peak control overhead. According to the result, the peak control overhead of SMART is lower than that of fast-OLSR by $10 \%$ on average. Furthermore, peak control overhead should be lower than the limit of network capacity for practical use. And this peak overhead of SMART seems acceptable from the view point of Wireless LAN networks' capacity.

Fig.7 shows the average packet delay. While SMART could maintain low delay, fast-OLSR caused unacceptable packet delay for voice communication because packet retransmission frequently occurred due to collisions. The delay of SMART is lower than those of original OLSR with default and shorter intervals. This is the advantage of unidirectional movement notification mechanism. This mechanism reduces control message overhead so that packet retransmission due to collisions decreased.

We further evaluated the effect of link quality based routing. We compared SMART with and with- 


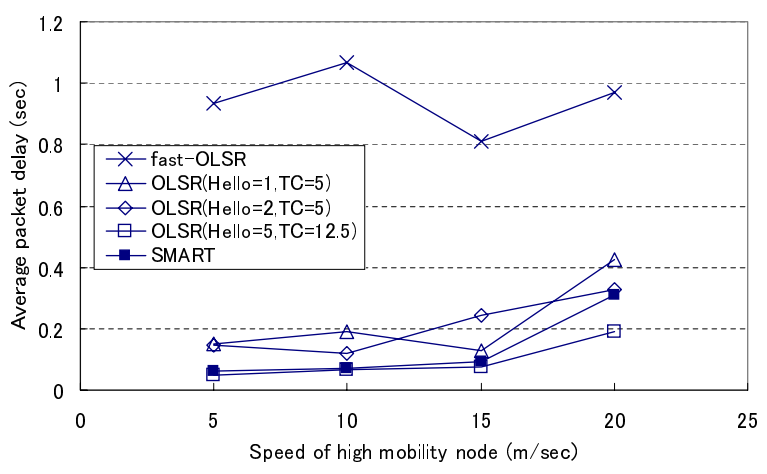

Fig. 7 Average packet delay

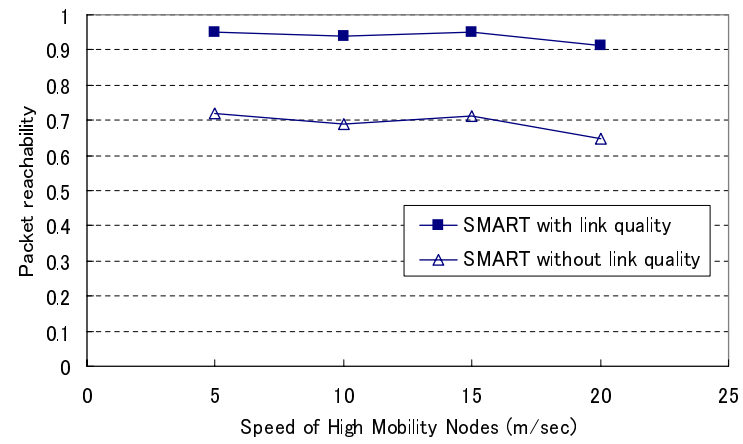

Fig. 8 Effect of link quality based routing on packet reachability

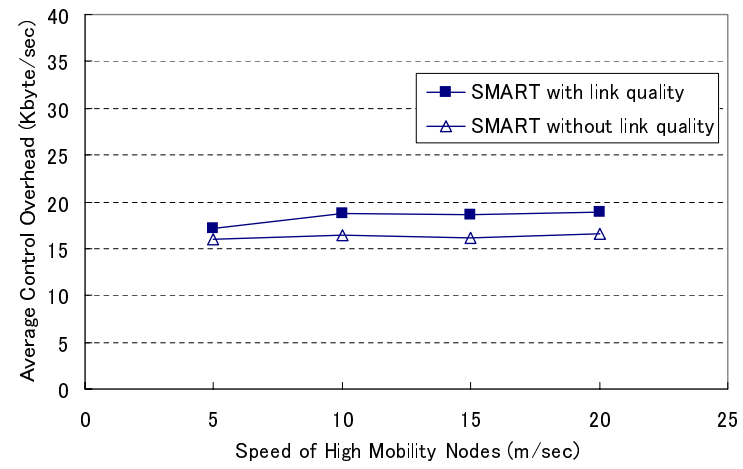

Fig. 9 Effect of link quality based routing on average control overhead

out the mechanism of link quality based routing. Fig.8 and Fig.9 show packet reachability and average control overhead, respectively. According to these results, link quality based routing improved packet reachability by $20 \%$ with a $10 \%$ increase in overhead. Fig.10 shows packet delay. This result reveals that 1) link quality based route calculation has little effect on packet delay, and 2) UMN can contribute to keep packet delay low.

Unlike original OLSR, the more fast moving nodes exist, the more control overhead of SMART increases.

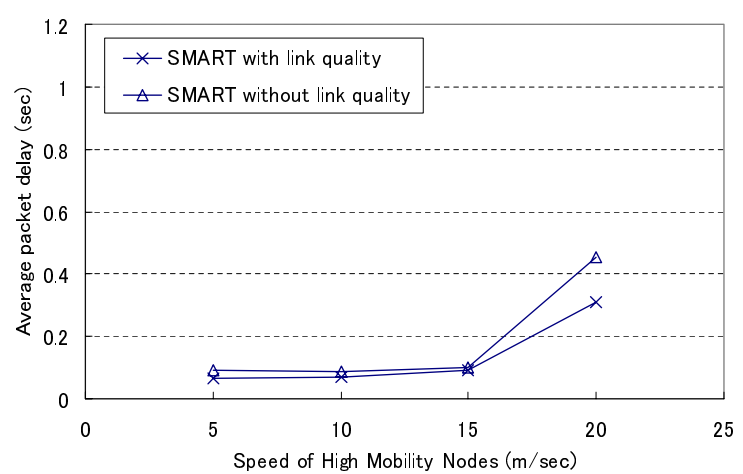

Fig. 10 Effect of link quality based routing on packet delay

Thus, the number of high mobility nodes affects the performance of SMART. Since SMART focuses on the use for a rescue operation in a disaster area, it is important to know its limitation from practical view points. To evaluate the limitation of SMART, we have compared the performance of SMART, fast-OLSR and original OLSR by changing the number of high mobility node. In the experiments, the number of high mobility nodes were changed from 5 to 20 . Other parameters were same as described in 4.2. The simulation results of reachability, average delay and average control overhead are shown in Fig.11, Fig.12 and Fig.13 respectively.

The reachability of fast-OLSR is degraded rapidly as the number of high mobility nodes increases. SMART can always achieve better packet reachability than fast-OLSR and original OLSR. However, the packet delay of SMART got worse when 20 high mobility nodes exist. The control overhead of SMART exceeded that of original OLSR with over 15 high mobility nodes. According to these results, it is supposed that SMART can achieve better communication quality in networks in which $15 \%$ of total nodes are high mobility nodes. If the percentage of high mobility nodes is more than $20 \%$, homogeneous aspect of network becomes apparent and exceeds beyond the support of SMART. Original OLSR is sufficient for such environment.

\section{Conclusion}

In this paper, we proposed Scalable Mobility Adaptive Routing Techniques (SMART) for mobile ad hoc networks with heterogeneous mobility. SMART introduces several mechanisms based on OLSR to improve communication quality with low control overhead. The main ideas in SMART are the unidirectional movement notification and the link-quality based route calculation.

- The unidirectional movement notification reduces total control overhead by the combination of indirect link break detection mechanism and new message generation mechanism. It reduces packet col- 


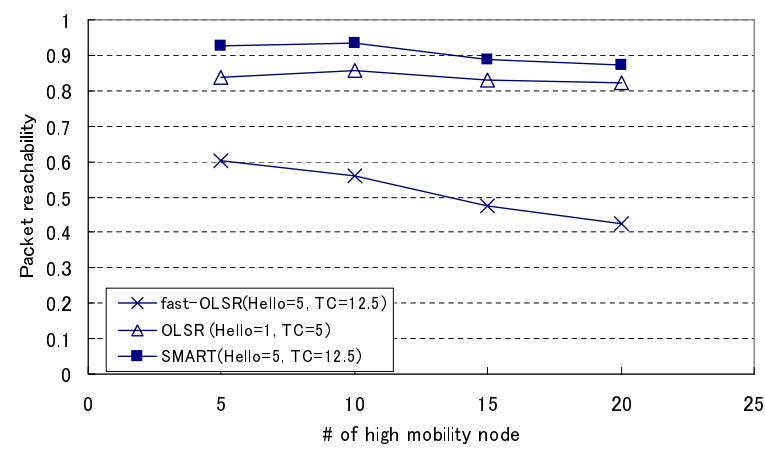

Fig. 11 Packet reachability vs. number of high mobility node

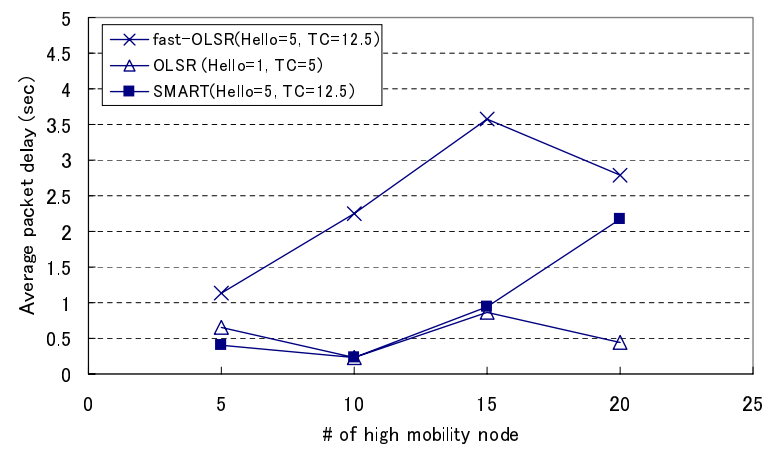

Fig. 12 Packet delay vs. number of high mobility node

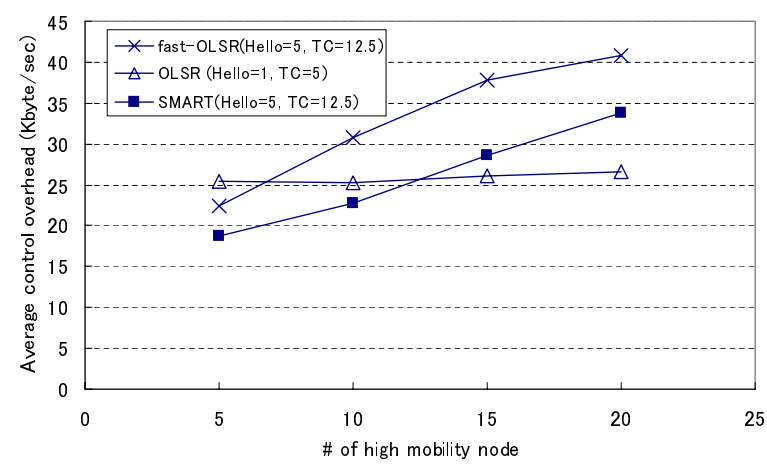

Fig. 13 Average control overhead vs. number of high mobility node

lision and contributes to low packet delay.

- The link quality based route calculation enables a node to switch to a better route before a current route breaks, and contributes to high packet reachability.

In our simulations which assume the real rescue operation, SMART showed its performance to improve packet reachability with low control overhead compared with other existing schemes. The experimental results also showed its ability to keep packet delay low. While
SMART can improve communication quality in our simulations, the performance evaluation of SMART in actual fields remains as a future work.

\section{References}

[1] M. Abolhasan, T. Wysocki, and E. A. Dutkiewicz, A review of routing protocols for mobile ad hoc networks, Ad Hoc Networks, vol.2, no.1, pp.1-22, Jan. 2004

[2] X. Hong, K. Xu, and M. Gerla, Scalable Routing Protocols for Mobile Ad Hoc Networks, IEEE Network Mag., Jul. 2002

[3] E. M. Royer and C.-K. Toh, A Review of Current Routing Protocols for Ad-Hoc Mobile Wireless Networks, IEEE Pers. Commun., Apr. 1999

[4] G. Malkin, RIP version 2, IETF RFC2453, November 1998

[5] J. Moy, OSPF version 2, IETF RFC2328, April 1998

[6] T. Clausen, P. Jacquet, A. Laouiti, P. Muhlethaler, a. Qayyum and L. Viennot, Optimized Link State Routing Protocol for Ad Hoc Networks, IEEE INMIC Pakistan 2001

[7] T. Clausen and P. Jacquet, Optimized Link State Routing Protocol (OLSR), IETF RFC3626, Oct. 2003

[8] Internet Engineering Task Force MANET-WG Web Page, http://www.ietf.org/html.charters/manet-charter.html

[9] M. Benzaid, P. Minet, K. Al Agha, Integrating fast mobility in the OLSR routing protocol, Fourth IEEE Conference on Mobile and Wireless Communications Networks (MWCN), Stockholm Sweden, Sep. 2002

[10] M. Benzaid, P. Minet, K. Al Agha, Analysis and simulation of Fast-OLSR, Proc. the 57th IEEE Semiannual Vehicular Technology Conference (VTC), Jeju Korea, April 2003

[11] K. Pahlavan, P. Krishnamurphy, A. Hatami, M. Ylianttila, J. Makela, R. Pichana and J. Vallstrom, Handoff in Hybrid Mobile Data Network, IEEE Pers. Comm. vol.7 Issue 2 pp. 34-47, Apr. 2000

[12] R. Ogier, F. Templin, and M. Lewis, Topology Dissemination based on Reverse Path Forwarding (TBRPF), IETF RFC3684, Feb. 2004

[13] G. Pei, M. Gerla, X. Hong and C. C. Chiang, A Wireless Hierarchical Routing Protocol with Group Mobility, Proc. IEEE WCNC'99, New Orleans, LA, Sep. 1999

[14] M. Gerla, X. Hong and G. Pei, Landmark Routing for Large Ad Hoc Wireless Networks, Proc. IEEE GLOBECOM 2000, San Francisco, CA, Nov. 2000

[15] Fire and Disaster Management Agency Japan, Activity report of International Rescue Team in Taiwan earthquake disaster (in Japanese), http://www.fdma.go.jp/ugoki/1999_11.02.pdf, Nov. 1999

[16] The network simulator ns2, http://www.isi.edu/nsnam/ns/

[17] OOLSR - Object oriented OLSR, http://hipercom.inria.fr/OOLSR/ 


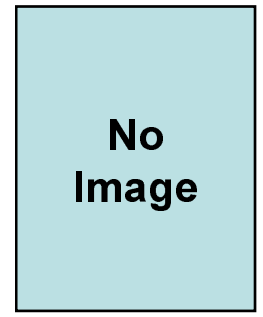

Tomohiko Yagyu received the B.E. and M.E. degree from Osaka University, Japan, in 1993 and 1995, respectively. He joined NEC Corporation in 1995. He is also a doctoral candidate at Tsukuba University since 2006. His current research interest is reliability and robustness enhancement for wired and wireless networks. He is a member of IPSJ and IEEE.

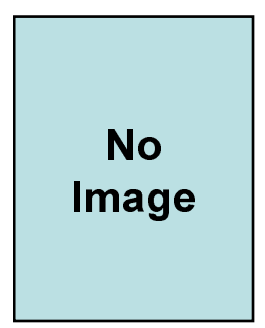

Masahiro Jibiki received the Ph.D. degree in systems management from the University of Tsukuba, Tokyo, Japan. He is currently a Researcher in Central Research Laboratories, NEC Corporation, and is also Visiting Professor in the University of Wakayama, Japan, from 2006. His research interests include networking, distributed system and software science.

Kenichi Yoshida received his $\mathrm{PhD}$ from Osaka University in '92. In '80, he joined Hitachi Ltd, and is working for University of Tsukuba from '02. His current research interest includes application of internet and application of machine learning techniques. 